\title{
Angular distribution of leptons from the decay of massive vector bosons
}

\author{
Edmond L. Berger ${ }^{1}$, Jian-Wei Qiu ${ }^{1,2}$, and Ricardo A. Rodriguez-Pedraza ${ }^{2}$ \\ ${ }^{1}$ High Energy Physics Division, Argonne National Laboratory, Argonne, IL 60439, U.S.A. \\ ${ }^{2}$ Department of Physics and Astronomy, Iowa State University, Ames, IA 50011, U.S.A.
}

(Dated: August 30, 2007)

\begin{abstract}
We examine the transverse momentum $Q_{\perp}$ dependence of the helicity structure functions for massive vector bosons of mass $Q$ in hadron reactions. We demonstrate that large logarithmic terms of the form $\ln \left(Q / Q_{\perp}\right)$ in the helicity structure functions have the same origin as the logarithmic terms in the angular-integrated cross section and that they can be resummed to all orders in the strong coupling $\alpha_{s}$, in the same way as the angular-integrated cross section. As a consequence of current conservation, the resummed part of the helicity structure functions preserves the Lam-Tung relation as a function of $Q_{\perp}$ to all orders in $\alpha_{s}$.
\end{abstract}

Introduction Massive vector boson production in hadronic interactions is a potent probe of short-distance dynamics in strong and electroweak interactions. The production of massive virtual photons, the Drell-Yan process 1], tests predictions of perturbative quantum chromodynamics (QCD) at high orders in perturbation theory and constrains parton distributions. Production of the intermediate weak vector bosons, the $W$ and $Z$, supplies accurate measurements of the masses of these bosons, and, particularly in the case of the $W$ boson, provides electroweak observables that bound the mass of the Higgs boson within the framework of the standard model of particle physics [2].

The massive virtual photon, the $W$ boson, and the $Z$ boson all have important decay modes into pairs of leptons. The angular distribution of these leptons, measured in the rest-frame of the parent states, determines the alignment (polarization) of the massive vector boson and, consequently, more precise information on the production dynamics than is accessible from the spinaveraged rate alone. An understanding of the changes expected in the angular distribution as a function of the transverse momentum $Q_{\perp}$ is a topic of considerable importance, both for refined tests of QCD and for reduced systematic uncertainties on the determination of the $\mathrm{W}$ boson mass [3, 4]. In this Letter, we derive predictions of the angular distribution as a function of $Q_{\perp}$, including resummation to all orders in the strong coupling strength $\alpha_{s}$ of the effects of initial state soft gluon radiation.

The general formalism for the description of the angular distribution in terms of helicity structure functions is developed for the Drell-Yan process in Ref. [5]. The cross section may be expressed as

$$
\begin{aligned}
\frac{d \sigma}{d^{4} q d \Omega}= & \frac{\alpha_{\mathrm{em}}^{2}}{2(2 \pi)^{4} S^{2} Q^{2}}\left[W_{T}\left(1+\cos ^{2} \theta\right)\right. \\
& +W_{L}\left(1-\cos ^{2} \theta\right)+W_{\Delta}(\sin (2 \theta) \cos \phi) \\
& \left.+W_{\Delta \Delta}\left(\sin ^{2} \theta \cos (2 \phi)\right)\right],
\end{aligned}
$$

where $q$ denotes the four-vector momentum of the massive virtual photon. The variables $\theta$ and $\phi$ are the polar and azimuthal angles in the virtual photon rest frame, and $\Omega$ is the solid angle $\Omega(\theta, \phi)$. The four independent helicity structure functions $W_{i}$ depend on the virtual photon's mass $Q$, transverse momentum $Q_{\perp}$, and rapidity $y$, as well as on the center-of-mass energy $\sqrt{S}$ of the production process. The dependence of the four $W_{i}$ on $Q_{\perp}$ is our central focus in this Letter.

At the most basic level, a massive virtual photon arises from quark-antiquark annihilation $q+\bar{q} \rightarrow \gamma^{*}$ in a collision of hadrons, and it is produced with $Q_{\perp}=0$ within the framework of collinear factorization in perturbative QCD [6]. First-order gluon radiation supplies finite $Q_{\perp}$, through the quark-antiquark and quark-gluon subprocesses, $q+\bar{q} \rightarrow \gamma^{*}+g$ and $q+g \rightarrow \gamma^{*}+q$. These finite-order subprocesses yield singular differential cross sections as a function of $Q_{\perp}$ in the limit $Q_{\perp} \rightarrow 0$. For the angular-integrated cross section, $d \sigma / d^{4} q$, it is well established that this divergence can be removed after resummation of the singular terms from initial-state gluon emission to all orders in $\alpha_{s}$ [7, 8]. In this Letter, we examine in detail the structure of the singular contributions for each helicity structure function $W_{i}$. We show explicitly that the $Q_{\perp} \rightarrow 0$ singular contributions to $W_{T}, W_{L}$, and $W_{\Delta \Delta}$ have the same origin as that of the angular integrated cross section and that they can be resummed to all orders in $\alpha_{s}$.

Asymptotic tensor In this section, we extract the perturbatively singular expressions for each term in the Drell-Yan hadronic tensor, and we introduce an asymptotic tensor that is defined to include all the singular terms and, in addition, to conserve the electromagnetic current.

The full Drell-Yan hadronic tensor can be written as [5]

$$
\begin{aligned}
W^{\mu \nu}= & -\left(g^{\mu \nu}-T^{\mu} T^{\nu}\right)\left(W_{T}+W_{\Delta \Delta}\right) \\
& -2 X^{\mu} X^{\nu} W_{\Delta \Delta}+Z^{\mu} Z^{\nu}\left(W_{L}-W_{T}-W_{\Delta \Delta}\right) \\
& -\left(X^{\mu} Z^{\nu}+X^{\nu} Z^{\mu}\right) W_{\Delta}
\end{aligned}
$$

where $T^{\mu}=q^{\mu} / Q$, and $X^{\mu}, Y^{\mu}$, and $Z^{\mu}$ are orthogonal unit vectors in the virtual photon's rest frame, with $T^{2}=1$, and $X^{2}=Y^{2}=Z^{2}=-1$. Different choices of the axes lead to different $\vec{q}=0$ frames $[5]$. In the Collins- 
Soper frame [9], all perturbatively calculated helicity structure functions at order $\alpha_{s}$ and beyond are singular as $Q_{\perp} / Q \rightarrow 0: W_{T}$ and $W_{\Delta}$ have the power divergences, $Q^{2} / Q_{\perp}^{2}$ and $Q / Q_{\perp}$, respectively, as well as $\ln \left(Q / Q_{\perp}\right)$ divergences, while $W_{L}$ and $W_{\Delta \Delta}$ show only $\ln \left(Q / Q_{\perp}\right)$ divergences [10, 11]. By expanding the full Drell-Yan hadronic tensor in Eq. (2) in the limit $Q_{\perp} / Q \rightarrow 0$, we obtain the following form for its singular terms [12, 13]

$$
\begin{aligned}
W_{\text {Sing }}^{\mu \nu}=( & \left.-g^{\mu \nu}+\bar{n}^{\mu} n^{\nu}+n^{\mu} \bar{n}^{\nu}\right) W_{2}^{\text {Asym }} \\
+ & \frac{1}{\sqrt{2}}\left[\frac{Q_{\perp}}{Q}\left(n_{\perp}^{\mu} \bar{n}^{\nu}+\bar{n}^{\mu} n_{\perp}^{\nu}\right) \mathrm{e}^{y}\right] \\
& \times\left(W_{2}^{\text {Asym }}-\frac{Q}{Q_{\perp}} W_{1}^{\text {Asym }}\right) \\
+ & \frac{1}{\sqrt{2}}\left[\frac{Q_{\perp}}{Q}\left(n_{\perp}^{\mu} n^{\nu}+n^{\mu} n_{\perp}^{\nu}\right) \mathrm{e}^{-y}\right] \\
& \times\left(W_{2}^{\text {Asym }}+\frac{Q}{Q_{\perp}} W_{1}^{\text {Asym }}\right)
\end{aligned}
$$

with two asymptotically divergent scalar functions: $W_{2}^{\text {Asym }} \propto Q^{2} / Q_{\perp}^{2}$ and $W_{1}^{\text {Asym }} \propto Q / Q_{\perp}$ as $Q_{\perp} / Q \rightarrow 0$. In Eq. (3), $\bar{n}^{\mu}=\delta^{\mu+}, n^{\mu}=\delta^{\mu-}$, and $n_{\perp}^{\mu}=\delta^{\mu \perp}$ are unit vectors that specify the light-cone coordinates of the laboratory frame, with $n^{2}=\bar{n}^{2}=0, n_{\perp}^{2}=-1, n \cdot \bar{n}=1$, and $n_{\perp} \cdot n=n_{\perp} \cdot \bar{n}=0$. In this frame, the incoming hadron momenta are $P_{1}^{\mu}=\sqrt{S / 2} \bar{n}^{\mu}$ and $P_{2}^{\mu}=\sqrt{S / 2} n^{\mu}$, respectively, and the virtual photon momentum: $q^{\mu}=$ $Q^{+} \bar{n}^{\mu}+Q^{-} n^{\mu}+Q_{\perp} n_{\perp}^{\mu}$, with $Q^{+}=\sqrt{\left(Q^{2}+Q_{\perp}^{2}\right) / 2} e^{y}$ and $Q^{-}=\sqrt{\left(Q^{2}+Q_{\perp}^{2}\right) / 2} e^{-y}$.

The singular tensor as $Q_{\perp} / Q \rightarrow 0$ in Eq. (3) is not current conserving since $q_{\mu} W_{\text {Sing }}^{\mu \nu} \neq 0$. In order to resum the singular terms of the hadronic tensor to all orders in $\alpha_{s}$, we require a tensor that includes all the singular terms and also conserves the current perturbatively at any order of $\alpha_{s}$. We use the term asymptotic tensor for this current-conserving tensor. We define it to be

$$
\begin{aligned}
W_{\text {Asym }}^{\mu \nu}=( & \left.-g^{\mu \nu}+\bar{n}^{\mu} n^{\nu}+n^{\mu} \bar{n}^{\nu}\right) W_{2}^{\text {Asym }} \\
+ & \frac{Q_{\perp}}{Q^{-}}\left(n_{\perp}^{\mu} \bar{n}^{\nu}+\bar{n}^{\mu} n_{\perp}^{\nu}+\frac{Q_{\perp}}{Q^{-}} \bar{n}^{\mu} \bar{n}^{\nu}\right) \\
& \times \frac{1}{2}\left[W_{2}^{\mathrm{Asym}}-\frac{Q}{Q_{\perp}} W_{1}^{\mathrm{Asym}}\right] \\
+ & \frac{Q_{\perp}}{Q^{+}}\left(n_{\perp}^{\mu} n^{\nu}+n^{\mu} n_{\perp}^{\nu}+\frac{Q_{\perp}}{Q^{+}} n^{\mu} n^{\nu}\right) \\
& \times \frac{1}{2}\left[W_{2}^{\mathrm{Asym}}+\frac{Q}{Q_{\perp}} W_{1}^{\mathrm{Asym}}\right] .
\end{aligned}
$$

It is equal to the singular tensor in Eq. (3) plus a minimal non-singular term such that $q_{\mu} W_{\mathrm{Asym}}^{\mu \nu}=0$.

The angular-integrated Drell-Yan cross section is obtained from the trace, $d \sigma / d^{4} q \propto-g_{\mu \nu} W^{\mu \nu}$. The trace of the asymptotic tensor in Eq. (4) should therefore be fixed by the asymptotic term $W^{\text {Asym }}$ in the angular-integrated Drell-Yan transverse momentum distribution [8]. This statement allows us to fix uniquely the asymptotically divergent function $W_{2}^{\text {Asym }}$ in Eq. (4): $W_{2}^{\text {Asym }}=W^{\text {Asym }} / 2$. However, the angular-integrated Drell-Yan cross section cannot fix the second scalar function $W_{1}^{\text {Asym }}$ in Eq. (4). This second function represents the singular perturbative behavior of the structure function $W_{\Delta}$. We can set this question aside for the purposes of the present Letter and concentrate on the resummation of the singular terms of the other three helicity structure functions, $W_{T}, W_{L}$, and $W_{\Delta \Delta}$.

We reexpress the asymptotic tensor in terms of the previously defined unit vectors in the Collins-Soper frame as

$$
\begin{aligned}
W_{\mathrm{Asym}}^{\mu \nu}= & {\left[\left(-g^{\mu \nu}+T^{\mu} T^{\nu}\right)-\frac{Q_{\perp}^{2} / Q^{2}}{1+Q_{\perp}^{2} / Q^{2}} X^{\mu} X^{\nu}\right.} \\
& \left.-\frac{1}{1+Q_{\perp}^{2} / Q^{2}} Z^{\mu} Z^{\nu}\right] \frac{W^{\mathrm{Asym}}}{2} \\
- & \frac{1}{1+Q_{\perp}^{2} / Q^{2}}\left[X^{\mu} Z^{\nu}+Z^{\mu} X^{\nu}\right] W_{1}^{\mathrm{Asym}}
\end{aligned}
$$

Upon comparison with Eq.(2), we immediately derive the corresponding asymptotic helicity structure functions,

$$
\begin{aligned}
W_{T}^{\text {Asym }} & =\left(1-\frac{1}{2} \frac{Q_{\perp}^{2} / Q^{2}}{1+Q_{\perp}^{2} / Q^{2}}\right) \frac{W^{\text {Asym }}}{2} \approx \frac{W^{\text {Asym }}}{2}, \\
W_{L}^{\text {Asym }} & =\frac{Q_{\perp}^{2} / Q^{2}}{1+Q_{\perp}^{2} / Q^{2}} \frac{W^{\text {Asym }}}{2} \approx \frac{Q_{\perp}^{2}}{Q^{2}} \frac{W^{\text {Asym }}}{2}, \\
W_{\Delta \Delta}^{\text {Asym }} & =\frac{1}{2} \frac{Q_{\perp}^{2} / Q^{2}}{1+Q_{\perp}^{2} / Q^{2}} \frac{W^{\text {Asym }}}{2} \approx \frac{1}{2} \frac{Q_{\perp}^{2}}{Q^{2}} \frac{W^{\text {Asym }}}{2} .
\end{aligned}
$$

Our first result is this derivation that the asymptotic part of these three helicity structure functions is proportional directly to the asymptotic part of the angular-integrated Drell-Yan transverse momentum distribution.

Perturbatively finite tensor We show in this section that the three asymptotic helicity structure functions derived in last section include all the divergent terms of the corresponding perturbatively calculated helicity structure functions, and therefore, that we can define a perturbatively finite tensor

$$
W_{\text {Finite }}^{\mu \nu} \equiv W_{\text {Pert }}^{\mu \nu}-W_{\text {Asym }}^{\mu \nu},
$$

at any order of $\alpha_{s}$. This perturbative finite tensor conserves the current since the asymptotic tensor conserves the current.

In terms of QCD collinear factorization [6], we can express the helicity structure functions in terms of partonlevel helicity structure functions, $w_{i}$ :

$$
W_{i}=\sum_{a b} \int \frac{d \xi_{1}}{\xi_{1}} \int \frac{d \xi_{2}}{\xi_{2}} \phi_{a}\left(\xi_{1}\right) \phi_{b}\left(\xi_{2}\right) w_{i}\left(\xi_{1}, \xi_{2}, q\right)
$$

with $i=T, L, \Delta \Delta$, and incoming parton distributions $\phi_{f}(\xi)$ of flavor $f$ and momentum fraction $\xi$. We have the same factorization relation for $W^{\text {Asym }}[8]$. 
For the spin-averaged $2 \rightarrow 2$ scattering processes $q \bar{q} \rightarrow$ $\gamma^{*} g$ and $q q \rightarrow \gamma^{*} q$, the asymptotic terms at the partonlevel are [13],

$$
\begin{aligned}
& \frac{w_{q \bar{q}}^{\text {Asym }}}{2} \approx e_{q}^{2} \frac{8 \pi^{2} \alpha_{s}}{3 x_{1} x_{2}} \frac{Q^{2}}{Q_{\perp}^{2}}\left\{P_{q q}\left(z_{2}\right) \delta\left(1-z_{1}\right)\right. \\
& +P_{q q}\left(z_{1}\right) \delta\left(1-z_{2}\right) \\
& \left.+2 C_{F} \delta\left(1-z_{1}\right) \delta\left(1-z_{2}\right)\left[\ln \left(\frac{Q^{2}}{Q_{\perp}^{2}}\right)-\frac{3}{2}\right]\right\} ; \\
& \frac{w_{q g}^{\text {Asym }}}{2} \approx e_{q}^{2} \frac{8 \pi^{2} \alpha_{s}}{3 x_{1} x_{2}} \frac{Q^{2}}{Q_{\perp}^{2}} P_{q g}\left(z_{2}\right) \delta\left(1-z_{1}\right),
\end{aligned}
$$

with $x_{1}=Q / \sqrt{S} e^{y}$ and $x_{2}=Q / \sqrt{S} e^{-y}$. The partonto-parton splitting functions are

$$
\begin{aligned}
P_{q q}(z) & =C_{F}\left[\frac{1+z^{2}}{(1-z)_{+}}+\frac{3}{2} \delta(1-z)\right], \\
P_{q g}(z) & =T_{R}\left[z^{2}+(1-z)^{2}\right],
\end{aligned}
$$

with $C_{F}=\left(N_{c}^{2}-1\right) /\left(2 N_{c}\right)=4 / 3, T_{R}=1 / 2$. Using Eq. (6) we find that as $Q_{\perp} / Q \rightarrow 0$, the partonic-level asymptotic terms in Eq. (9) remove all divergent contributions of the corresponding perturbatively calculated helicity structure functions. For the quark-antiquark annihilation subprocess,

$$
\begin{gathered}
w_{T}^{q \bar{q}}-\frac{w_{q \bar{q}}^{\text {Asym }}}{2} \Rightarrow \mathcal{O}\left(Q_{\perp}^{0}\right) \\
w_{L}^{q \bar{q}}-\frac{Q_{\perp}^{2}}{Q^{2}} \frac{w_{q \bar{q}}^{\text {Asym }}}{2} \Rightarrow \mathcal{O}\left(Q_{\perp}^{2}\right) \\
w_{\Delta \Delta}^{q \bar{q}}-\frac{1}{2} \frac{Q_{\perp}^{2}}{Q^{2}} \frac{w_{q \bar{q}}^{\text {Asym }}}{2} \Rightarrow \mathcal{O}\left(Q_{\perp}^{2}\right) .
\end{gathered}
$$

For the quark-gluon subprocess,

$$
\begin{aligned}
w_{T}^{q g}- & \frac{w_{q g}^{\mathrm{Asym}}}{2} \Rightarrow \mathcal{O}\left(Q_{\perp}^{0}\right) \\
w_{L}^{q g}- & \frac{Q_{\perp}^{2}}{Q^{2}} \frac{w_{q g}^{\mathrm{Asym}}}{2} \Rightarrow e_{q}^{2} \frac{8 \pi^{2} \alpha_{s}}{3 x_{1} x_{2}} \delta\left(1-z_{1}\right) \\
& \times\left[P_{q g}\left(-z_{2}\right)-P_{q g}\left(z_{2}\right)\right]+\mathcal{O}\left(Q_{\perp}^{2}\right) \\
w_{\Delta \Delta}^{q g}- & \frac{1}{2} \frac{Q_{\perp}^{2}}{Q^{2}} \frac{w_{q g}^{\mathrm{Asym}}}{2} \Rightarrow \frac{1}{2} e_{q}^{2} \frac{8 \pi^{2} \alpha_{s}}{3 x_{1} x_{2}} \delta\left(1-z_{1}\right) \\
& \times\left[P_{q g}\left(-z_{2}\right)-P_{q g}\left(z_{2}\right)\right]+\mathcal{O}\left(Q_{\perp}^{2}\right) .
\end{aligned}
$$

With $z_{1}$ and $z_{2}$ switched, Eq. (13) is also true for the gluon-quark subprocess. Other than the non-logarithmic finite piece (as $Q_{\perp} / Q \rightarrow 0$ ) in the quark-gluon contribution to $W_{L}$ and $W_{\Delta \Delta}$, the asymptotic tensor completely removes the leading term of the perturbatively calculated helicity structure functions as $Q_{\perp} / Q \rightarrow 0$.

We note that the Lam-Tung relation, $W_{L}=2 W_{\Delta \Delta}$, is obeyed by the singular terms of the perturbatively calculated helicity structure functions.
Polarized initial partons To gain insight into the uncanceled finite terms in $W_{L}$ and $W_{\Delta \Delta}$ for the $q g$ subprocess, we calculate the parton-level asymptotic and perturbatively finite helicity structure functions for "polarized" incoming parton states, defined as the states with incoming parton polarization projected onto the difference of the parton helicity states [13]. For the quark-antiquark subprocess, we find that $\Delta w_{q \bar{q}}^{\mathrm{Asym}}=w_{q \bar{q}}^{\mathrm{Asym}}$, whereas for the quark-gluon subprocess

$$
\frac{\Delta w_{q g}^{\text {Asym }}}{2} \approx e_{q}^{2} \frac{8 \pi^{2} \alpha_{s}}{3 x_{1} x_{2}} \frac{Q^{2}}{Q_{\perp}^{2}} \Delta P_{q g}\left(z_{2}\right) \delta\left(1-z_{1}\right),
$$

with

$$
\Delta P_{q g}(z)=T_{R}\left[z^{2}-(1-z)^{2}\right] .
$$

We note that $\Delta w_{q g}^{\text {Asym }}$ is different from $w_{q g}^{\text {Asym }}$ in Eq. (9). The finite contributions in the perturbatively computed parton-level helicity structure functions are

$$
\begin{aligned}
\Delta w_{T}^{q g}- & \frac{\Delta w_{q g}^{\mathrm{Asym}}}{2} \Rightarrow \mathcal{O}\left(Q_{\perp}^{0}\right) \\
\Delta w_{L}^{q g}- & \frac{Q_{\perp}^{2}}{Q^{2}} \frac{\Delta w_{q g}^{\mathrm{Asym}}}{2} \Rightarrow e_{q}^{2} \frac{8 \pi^{2} \alpha_{s}}{3 x_{1} x_{2}} \delta\left(1-z_{1}\right) \\
& \times\left[\Delta P_{q g}\left(-z_{2}\right)-\Delta P_{q g}\left(z_{2}\right)\right]+\mathcal{O}\left(Q_{\perp}^{2}\right) \\
\Delta w_{\Delta \Delta}^{q g}- & \frac{1}{2} \frac{Q_{\perp}^{2}}{Q^{2}} \frac{\Delta w_{q g}^{\mathrm{Asym}}}{2} \Rightarrow \frac{1}{2} e_{q}^{2} \frac{8 \pi^{2} \alpha_{s}}{3 x_{1} x_{2}} \delta\left(1-z_{1}\right) \\
& \times\left[\Delta P_{q g}\left(-z_{2}\right)-\Delta P_{q g}\left(z_{2}\right)\right]+\mathcal{O}\left(Q_{\perp}^{2}\right) .
\end{aligned}
$$

The uncanceled finite term in the helicity structure functions $W_{L}$ and $W_{\Delta \Delta}$ is proportional to

$$
P_{q g}\left(-z_{2}\right)-P_{q g}\left(z_{2}\right)=4 z_{2} T_{R},
$$

for unpolarized initial partonic states, and to

$$
\Delta P_{q g}\left(-z_{2}\right)-\Delta P_{q g}\left(z_{2}\right)=-4 z_{2} T_{R},
$$

for the "polarized" initial partonic states. Therefore, the uncanceled finite term in $W_{L}$ and $W_{\Delta \Delta}$ in the limit $Q_{\perp} / Q \rightarrow 0$ can also be removed by the asymptotic term if the incoming quark and gluon have the same helicity (both positive or negative). We also observe that, at this order, the uncanceled term in the quark-gluon subprocess is proportional to the helicity flipping splitting function,

$$
P_{q^{-} g^{+}}(z)=P_{q^{+} g^{-}}(z)=T_{R}(1-z)^{2} .
$$

The finite term as $Q_{\perp} / Q \rightarrow 0$ for the quark-antiquark subprocess at this order is removed completely by the asymptotic term since the helicity flipping splitting function for the quark vanishes at this order, $P_{q^{-} q^{+}}(z)=$ $P_{q^{+} q^{-}}(z)=0$.

Our second result in this Letter is that transverse momentum dependent factorization for the full hadronic tensor, which is the basis for the Collins-Soper-Sterman 
$b$-space resummation, holds at leading power in the $Q_{\perp} / Q$ expansion. Breaking of factorization occurs at subleading power but only in the helicity flipping channel and does not supply leading logarithmic terms.

Resummation As shown above, as $Q_{\perp} / Q \rightarrow 0$, all leading large logarithmic terms in the helicity structure functions $W_{T}, W_{L}$, and $W_{\Delta \Delta}$ are included in one asymptotic function, $W^{\text {Asym }}$, the same asymptotic function as in the resummed angular-integrated Drell-Yan cross section. Resummation of the large logarithmic terms of the Drell-Yan helicity structure functions can therefore be expressed in terms of the resummed contribution to the angular-integrated Drell-Yan cross section. Referring to Eq. (6), we obtain the resummed contribution to the helicity structure functions in the Collins-Soper frame as

$$
\begin{aligned}
W_{T}^{\text {Resum }} & =\left(1-\frac{1}{2} \frac{Q_{\perp}^{2} / Q^{2}}{1+Q_{\perp}^{2} / Q^{2}}\right) \frac{W^{\text {Resum }}}{2}, \\
W_{L}^{\text {Resum }} & =\frac{Q_{\perp}^{2} / Q^{2}}{1+Q_{\perp}^{2} / Q^{2}} \frac{W^{\text {Resum }}}{2}, \\
W_{\Delta \Delta}^{\text {Resum }} & =\frac{1}{2} \frac{Q_{\perp}^{2} / Q^{2}}{1+Q_{\perp}^{2} / Q^{2}} \frac{W^{\text {Resum }}}{2} .
\end{aligned}
$$

All depend on the same QCD resummed expression $W^{\text {Resum }}$ that pertains to the angular-integrated DrellYan cross section [8, 13]. We remark that resummation deals with the logarithmically divergent terms in the perturbatively calculated structure functions. There are remaining finite terms as defined in Eq. (7) that make up the full contribution to the structure functions.

Equation (20) shows that the Lam-Tung relation, $W_{L}=2 W_{\Delta \Delta}$, is satisfied by the resummed contribution to all orders in $\alpha_{s}$. This result is a direct consequence of current conservation that led us to the asymptotic tensor in Eq. (5). Furthermore, since the resummed contribution dominates the cross section in the region of small and intermediate values of $Q_{\perp}$, we expect that violation of the Lam-Tung relation as a function of $Q_{\perp}$ should be relatively small. This third result of our investigation is consistent with what is found in perturbative calculations at order $\alpha_{s}^{2}$ [14], but it is shown here to be true to all orders in $\alpha_{s}$. Data also show reasonable agreement with the Lam-Tung relation for moderate values of $Q_{\perp}$ [15].

Summary and Discussion In this Letter, we investigate the transverse momentum $Q_{\perp}$ dependence of the angular distribution of leptons from the decay of a massive virtual photon produced in hadron collisions. We work in terms of the four independent helicity structure functions that characterize this angular distribution. We show for the first time that the $Q_{\perp}$ dependence of the helicity structure functions $W_{T}, W_{L}$, and $W_{\Delta \Delta}$ is specified by the same all-orders resummed $Q_{\perp}$ distribution that characterizes the angular integrated cross section, up to $Q_{\perp}$ dependent kinematic factors that we list explicitly in Eq.(20). One consequence of this work is the demon- stration that the Lam-Tung relation, $W_{L}=2 W_{\Delta \Delta}$, between the longitudinal and the double-spin-flip structure functions is obeyed by the resummed cross sections as a function of $Q_{\perp}$ and that this relation is a direct result of the requirement of current conservation.

In this Letter, we work entirely in the collinear factorization approach and resum large logarithmic perturbative contributions when $Q_{\perp} / Q \rightarrow 0$. When $Q_{\perp}$ is very small, one might study the angular distribution in terms of transverse momentum dependent parton distributions [16].

In further work, we intend to examine the $Q_{\perp}$ dependence of $W$ and $Z$ boson production, where parity violating terms introduce additional helicity structure functions.

Acknowledgments E.L.B. is supported by the U. S. Department of Energy, Division of High Energy Physics, under Contract No. DE-AC-02-06CH11357. JW.Q. is supported in part by the U. S. Department of Energy under Grant No. DE-FG02-87ER40371 and in part by the Argonne University of Chicago Joint Theory Institute (JTI) Grant 03921-07-137. R.A.R. is supported in part by the U. S. Department of Energy under Grant No. DE-FG02-87ER40371. We are grateful to John T. Donohue, Bordeaux, for valuable communications.

[1] S. D. Drell and T. M. Yan, Phys. Rev. Lett. 25, 316 (1970) [Erratum-ibid. 25, 902 (1970)].

[2] T. Aaltonen et al. [CDF Collaboration], arXiv:0707.0085 [hep-ex], and references therein.

[3] C. Balazs, J. W. Qiu and C. P. Yuan, Phys. Lett. B 355, 548 (1995) arXiv:hep-ph/9505203.

[4] R. K. Ellis, D. A. Ross and S. Veseli, Nucl. Phys. B 503, 309 (1997) arXiv:hep-ph/9704239.

[5] C. S. Lam and W. K. Tung, Phys. Rev. D 18, 2447 (1978); Phys. Rev. D 21, 2712 (1980).

[6] J.C. Collins, D.E. Soper, G. Sterman, Adv. Ser. Direct. High Energy Phys. 5, 1 (1988).

[7] Y. L. Dokshitzer, D. Diakonov and S. I. Troian, Phys. Rept. 58, 269 (1980).

[8] J. C. Collins, D. E. Soper and G. Sterman, Nucl. Phys. B 250, 199 (1985).

[9] J. C. Collins and D. E. Soper, Phys. Rev. D 16, 2219 (1977).

[10] J. C. Collins, Phys. Rev. Lett. 42, 291 (1979).

[11] D. Boer and W. Vogelsang, Phys. Rev. D 74, 014004 (2006) arXiv:hep-ph/0604177, and references therein.

[12] R. A. Rodriguez-Pedraza, Ph.D. dissertation, Iowa State University.

[13] E. L. Berger, J. W. Qiu, and R. A. Rodriguez-Pedraza, arXiv:0708.0578 [hep-ph], submitted to Physical Reveiw D.

[14] E. Mirkes, Nucl. Phys. B 387, 3 (1992); E. Mirkes and J. Ohnemus, Phys. Rev. D 51, 4891 (1995).

[15] L. Y. Zhu et al. [FNAL-E866/NuSea Collaboration], arXiv:hep-ex/0609005, and references therein.

[16] E. L. Berger, J. T. Donohue and S. Wolfram, Phys. Rev. 
D 17, 858 (1978); J. T. Donohue, in Moriond 1978, Proceedings, Phenomenology Of Quantum Chromodynamics,
Vol.I, Dreux 1978, 159-163. 